\title{
lonized calcium measurements during regional citrate anticoagulation in CRRT: we need better blood gas analyzers
}

\author{
Detlef Kindgen-Milles ${ }^{1}$, Marlies Ostermann ${ }^{2}$ and Torsten Slowinski ${ }^{3^{*}}$ (D) \\ See related research by Schwarzer et al., http://www.ccforum.com/content/19/1/321
}

Schwarzer et al. [1] showed discrepant postfilter ionized calcium concentrations [iCa] when using different blood gas analyzers (BGAs) and called for a change of the Fresenius regional citrate anticoagulation (RCA) protocol to ensure patient safety. Of note, precision of the BGAs was not tested.

In our opinion, the key message of their study is that commercially available BGAs are not accurate when measuring $[\mathrm{iCa}]$ outside the reference range and therefore clinicians should avoid using multiple BGAs to guide RCA in individual patients. However, there is no indication to change a RCA protocol which has been proven safe and effective in $>10$ studies including $>2000$ patients from different countries regardless of the BGA used [2-5].

Schwarzer et al. also raise concern about the potential risk of life-threatening citrate intoxication. Whether raised citrate levels are toxic or merely indicative of impaired cellular metabolism remains unclear, but excess citrate can cause metabolic alkalosis. The Fresenius RCA protocol and the technical specifications of the multifiltrate machine both include safety mechanisms to detect potential citrate accumulation early. The risk of citrate toxicity is low $(<3 \%)$, even in high-risk patients with liver failure $[3,5]$.

Given the proven advantages of RCA and the Kidney Disease Improving Global Outcomes (KDIGO) recommendation to use citrate as the first-line anticoagulant during continuous renal replacement therapy, the accuracy of commercially available BGA devices should be improved. In our opinion, there is no need to change a safe and effective protocol.

\section{Abbreviations}

[iCa]: Ionized calcium concentration; BGA: Blood gas analyzer; KDIGO: Kidney Disease Improving Global Outcomes; RCA: Regional citrate anticoagulation.

\section{Competing interests}

DKM, MO, and TS have received research grants and funds for speaking at symposia organized on behalf of Fresenius Medical Care, Germany.

\section{Authors' contributions}

All authors were responsible for analysis and interpretation of data, manuscript writing, and final approval of the manuscript.

\section{Author details}

${ }^{1}$ Department of Anesthesiology, University Hospital Düsseldorf, Heinrich-Heine-University, Moorenstr. 5, D-40225 Düsseldorf, Germany. 'Department of Critical Care, King's College London, Guy's and St. Thomas' Foundation Hospital, Westminster Bridge Road, London SE1 7EH, UK. ${ }^{3}$ Department of Nephrology, Charité Universitätsmedizin Berlin, Campus Mitte (CCM), Charitéplatz 1, D-10117 Berlin, Germany.

Published online: 09 December 2015

\section{References}

1. Schwarzer P, Kuhn S-O, Stracke S, Grundling M, Knigge S, et al. Discrepant post filter ionized calcium concentrations by common blood gas analyzers in CRRT using regional citrate anticoagulation. Crit Care. 2015;19:321.

2. Kalb R, Kram R, Morgera S, Slowinski T, Kindgen-Milles D. Regional citrate anticoagulation for high volume continuous venovenous hemodialysis in surgical patients with high bleeding risk. Ther Apher Dial. 2013;17:202-12.

3. Khadzhynov D, Schelter C, Lieker I, Mika A, Staeck O, et al. Incidence and outcome of metabolic disarrangements consistent with citrate accumulation in critically ill patients undergoing continuous venovenous hemodialysis with regional citrate anticoagulation. J Crit Care. 2014;29:265-71.

4. Morgera S, Schneider M, Slowinski T, Vargas-Hein O, Zuckermann-Becker H, et al. A safe citrate anticoagulation protocol with variable treatment efficacy and excellent control of the acid-base status. Crit Care Med. 2009;37:2018-24.

5. Slowinski T, Morgera S, Joannidis M, Henneberg T, Stocker R, et al. Safety and efficacy of regional citrate anticoagulation in continuous veno-venous hemodialysis in the presence of liver failure: The Liver Citrate Anticoagulation Threshold (L-CAT)—an observational study. Crit Care. 2015;19:349.

\footnotetext{
* Correspondence: torsten.slowinski@charite.de

${ }^{3}$ Department of Nephrology, Charité Universitätsmedizin Berlin, Campus

Mitte (CCM), Charitéplatz 1, D-10117 Berlin, Germany

Full list of author information is available at the end of the article
} 